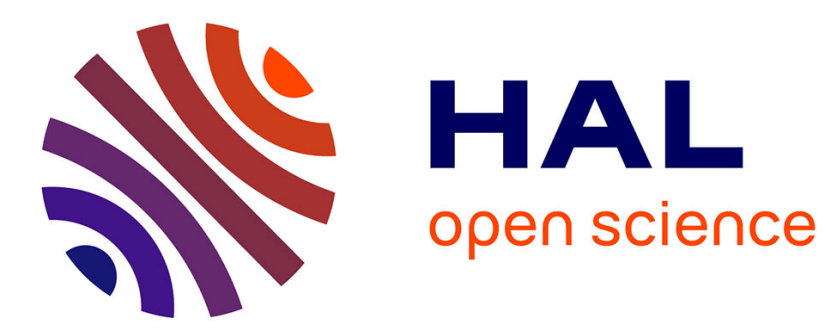

\title{
Bounded attitude control of a biomimetic flapping robot
} Hala Rifai, Nicolas Marchand, Guylaine Poulin

\section{To cite this version:}

Hala Rifai, Nicolas Marchand, Guylaine Poulin. Bounded attitude control of a biomimetic flapping robot. ROBIO 2007 - IEEE International Conference on Robotics and Biomimetics, 2007, Sanya, China. 10.1109/ROBIO.2007.4522125 . hal-00175970

\section{HAL Id: hal-00175970 https://hal.science/hal-00175970}

Submitted on 26 Apr 2018

HAL is a multi-disciplinary open access archive for the deposit and dissemination of scientific research documents, whether they are published or not. The documents may come from teaching and research institutions in France or abroad, or from public or private research centers.
L'archive ouverte pluridisciplinaire HAL, est destinée au dépôt et à la diffusion de documents scientifiques de niveau recherche, publiés ou non, émanant des établissements d'enseignement et de recherche français ou étrangers, des laboratoires publics ou privés. 


\section{Bounded attitude control of a biomimetic flapping robot}

\author{
Hala Rifaï and Nicolas Marchand \\ GIPSA-lab, Control Systems Department \\ UMR CNRS 5216-INPG-UJF, ENSIEG BP 46 \\ 38402 Saint Martin d'Hères Cedex, FRANCE \\ \{Hala.Rifai, Nicolas.Marchand\}@gipsa-lab.inpg.fr
}

\author{
Guylaine Poulin \\ G2E-lab \\ UMR CNRS 5269-INPG-UJF, ENSIEG BP 46 \\ 38402 Saint Martin d'Hères Cedex, FRANCE \\ Guylaine.Poulin@g2elab.inpg.fr
}

\begin{abstract}
Flapping micro Unmanned Air Vehicles (UAVs) can be considered as high frequency periodic systems. Based on the averaging theory, only the mean aerodynamic forces and torques over a wingbeat affect the movement of the body. Therefore, a nonlinear bounded state feedback control law is calculated upon the averaged model and applied to the time varying system represented by the flapping body in order to stabilize its attitude.
\end{abstract}

Index Terms-Biomimetic flight, modeling, averaging, bounded attitude control.

\section{INTRODUCTION}

Biomimetic robotics has become a growing reality that will revolutionize even more the industrial world in the near future considering the greater maneuverability, robustness in performance and energy efficiency that animals present. For instance, fish need up to $70 \%$ less energy than underwater vehicles to move the same weight at the same speed [1]. Another class of biomimetic robots is constituted by flapping micro UAVs that have recently emerged, combining the advantages of rotary and fixed airfoils. Their theoretical high maneuverability makes possible the execution of punctual tasks in narrow places. Moreover, the flapping flight presents an efficiency in lift production, energy consumption and noise reduction. On the other hand, although considerable effort has been exploited to understand the complex structure and behavior of insects, still little is known about mechanisms deployed during complex maneuvers [2]. Researches in this domain attract biology, aeronautic and robotic societies. The most important project is the Micromechanical Flying Insect (MFI) supported by the DARPA [3], [4]. Other projects are held all over the world, "Volo Libero" (University of Toronto, Canada), "NASA Solar Flapper" (NASA, USA), "Delfly" (Delft University of Technology, Netherlands), "Remanta" (ONERA, France), "OVMI"1 (France), etc. The progress in microelectronic technologies, materials, sensors, actuators, embedded dedicated computation abilities, communication tools, etc. helped the feasibility and development of these aircrafts. Micro flapping UAVs can be used for many indoor

\footnotetext{
${ }^{1}$ The OVMI project is supported by the French National Research Agency (ANR). It involves the IEMN (Valenciennes, Lille - France) for microelectronic study, the ONERA (Palaiseau - France) for fluid mechanics modeling, the SATIE (Cachan - France) for energy aspects, the GIPSA-lab (Grenoble - France) for modeling and control and the G2E-lab (Grenoble - France) for centimetric prototypes.
}

and outdoor civil applications (monitoring, inspecting monuments, intervening in narrow and dangerous environments) or for military applications where the biomimetic behavior helps achieving discrete missions (spying and investigating unknown areas) [5].

The work in this paper takes part of the "OVMI" projet $^{1}$, aiming to design and develop a flying object (see Fig.1), capable of sustained autonomous flight, by mimicking the insects in performance and size.

The present paper deals with the problem of attitude control of a flapping body. Few previous works have treated this problem. In [5], only the stabilization of the pitch angle using sliding mode control is presented. Others have worked on this problem using the linearized dynamics of the system to compute Proportional Derivative (PD) [6], state feedback controllers [7], [8] that can be based on pole placement [9] and Linear Quadratic Gaussian (LQG) optimal control [4], [6].

One particularity of the present work is that the attitude stabilization takes into account the saturation of the actuators through a bounded state feedback nonlinear control law. Another particularity is that the orientation of the flapping body is determined by quaternion [10], preventing numerical singularities induced by Euler angles.

The paper is organized as follows. Section II proposes a simplified model and wings parametrization of a flapping micro UAV and its average behavior over a single wingbeat period. In section III, a control law is proposed and its convergence is proven, based on rigid bodies model and averaging theory. Section IV shows the results of some simulations and the robustness of the control law. Finally, section V gives some conclusions and introduces future works.

\section{FLAPPING FLIGHT MODELING}

A flapping micro UAV is composed mainly of flapping wings and a rigid body. A simplified model based on lim- 
ited wings' kinematics parametrization is proposed, aiming to compute control laws adaptable for implementation on embedded processors having low computational capabilities.

\section{A. Wings trajectory parametrization}

Each wing is considered as a rigid body having three main degrees of freedom: flapping, lagging and feathering, then associated to a three sided frame $\mathbb{R}^{w}(\vec{r}, \vec{t}, \vec{n}, \psi, \phi, \theta)$ (see Fig. 2). Flapping is an up and down movement of the wing, movement represented by a rotation of angle $\phi$ about axis $\vec{t}$ parallel to the wing chord, oriented from trailing to leading edge. Feathering is a twist motion of the wing around its base axis, in order to change its angle of attack, represented by a rotation of angle $\psi$ about axis $\vec{r}$ oriented from the wing base to its tip. Lagging is a forward and backward movement of the wing parallel to the micro UAV's body, it is a rotation of angle $\theta$ about axis $\vec{n}$ perpendicular to the wing plane oriented so that the three-sided frame $(\vec{r}, \vec{t}, \vec{n})$ is direct. Flapping is considered as the translational movement of the wing and feathering as the rotational one. Lagging is not taken into account in this work $(\theta=0)$ in order to use actuators for 2 DOF only. Moreover, the pitch torque, that can be generated by $\theta$ 's variation, is finally created by some added mass moving inside the body. As in [11], angles $\phi$ and $\psi$ are assumed to vary according to saw tooth and pulse functions respectively, so that the wing changes its orientation at the end of each half stroke (see Fig. 3).
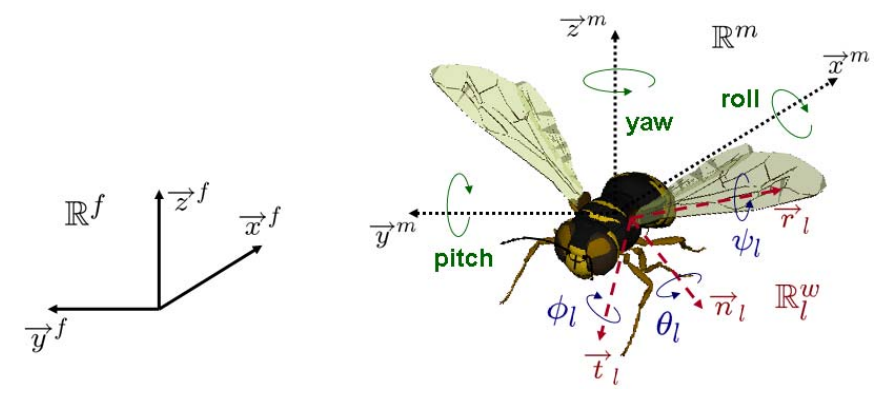

Fig. 2. Frames and wings angles

$$
\begin{aligned}
& \phi(t)=\left\{\begin{array}{lr}
\phi_{0}\left(1-\frac{2 t}{\kappa T}\right) & 0 \leq t \leq \kappa T \\
\phi_{0}\left(2 \frac{t-\kappa T}{(1-\kappa) T}-1\right) & \kappa T<t \leq T
\end{array}\right. \\
& \psi(t)=\psi_{0} \operatorname{sign}(\kappa T-t) \quad 0 \leq t \leq T \\
& \theta(t)=0 \quad 0 \leq t \leq T
\end{aligned}
$$

where sign designates the classical sign function, $T$ is the wingbeat period, $\kappa$ is the ratio of downstroke duration to total wingbeat period, $\phi_{0}$ and $\psi_{0}$ are respectively the amplitudes of stroke and rotation angles. These two last parameters considered for both left and right wings, will be taken as control variables, as explained in the following.

\section{B. Body's dynamics model}

The simplified body's model developed in this work uses quaternion [10] to represent the orientation of the flapping
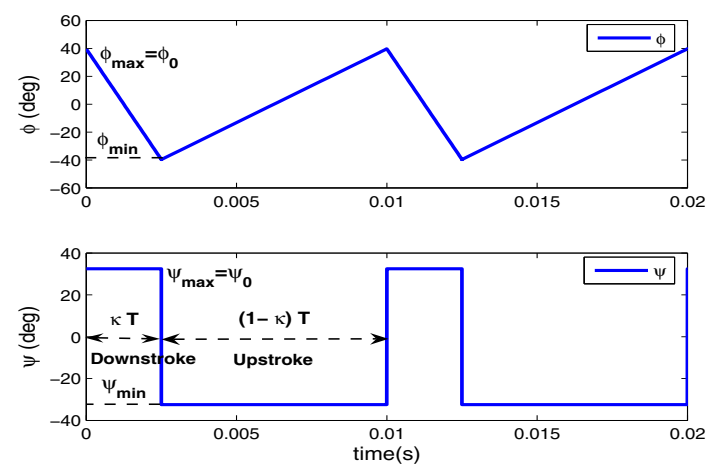

Fig. 3. Wing angles configuration over two wingbeat periods: stroke angle $\phi$ and rotation angle $\psi$

body. The quaternion is a four elements vector consisting of a unit vector $\vec{e}$, known as Euler axis, and a rotation angle $\nu$ about this axis

$$
q=\left[\cos \frac{\nu}{2}\left(\vec{e} \sin \frac{\nu}{2}\right)\right]^{T}=\left[\begin{array}{ll}
q_{0} & \vec{q}^{T}
\end{array}\right]^{T}
$$

where $q_{0} \in \mathbb{R}$ is the scalar part and $\vec{q}=\left[\begin{array}{lll}q_{1} & q_{2} & q_{3}\end{array}\right]^{T} \in$ $\mathbb{R}^{3}$ the vector part of the quaternion. $q \in \mathbb{H}$ where $\mathbb{H}=\left\{q \mid q_{0}^{2}+\vec{q}^{T} \vec{q}=1\right\}$ is the Hamilton space. The quaternion represents a rotation from the fixed frame $\mathbb{R}^{f}$ to the mobile frame $\mathbb{R}^{m}$ attached to the insect's center of gravity (see Fig. 2). The rotation matrix is defined by

$$
R(q)=\left(q_{0}^{2}-\vec{q}^{T} \vec{q}\right) I_{3}+2\left(\vec{q} \vec{q}^{T}-q_{0} \hat{q}^{T}\right)
$$

where $I_{3}$ is the identity matrix and $\hat{q}$ is the skew symmetric tensor associated to $\vec{q}$

$$
\hat{q}=\left[\begin{array}{ccc}
0 & q_{3} & -q_{2} \\
-q_{3} & 0 & q_{1} \\
q_{2} & -q_{1} & 0
\end{array}\right]
$$

The error between the current and desired orientations of the body, respectively denoted as $q$ and $q_{d}$, is quantified by the quaternion error: $q_{e}=q \otimes q_{d}^{-1}$, where $q^{-1}$ is the quaternion conjugate given by $q^{-1}=\left[q_{0}-\vec{q}^{T}\right]^{T}, \otimes$ is the quaternion product defined by $q \otimes Q=\left[\left(q_{0} Q_{0}-\vec{q} \vec{Q}\right)\left(q_{0} \vec{Q}+Q_{0} \vec{q}+\vec{q} \wedge \vec{Q}\right)^{T}\right]^{T}$, and $\wedge$ denotes the vectorial product.

The position and attitude dynamics of a flapping micro UAV are due to the external forces and torques applied to the body. Flight theory is based on:

- The aerodynamic forces, which horizontal component, the thrust, makes the UAV maintain a constant velocity or accelerate, and vertical component, the lift, balances the weight of the UAV and allows ascending movements.

- The drag that represents the body's damping forces.

- The weight that represents the gravitational forces.

Aerodynamic forces are based on three major mechanisms [12]: delayed stall, rotational lift and wake capture. The first one is developed during the translational movement of the wing, while the last two ones are developed during the 
rotation of the wing and have minor contribution to the totality of the aerodynamic forces [11]. Therefore, these two effects are neglected in this work. The wings' inertia mainly leads to wings camber reversal at the beginning of each half stroke. This phenomenon is explained and numerically considered afterwards through the force's aerodynamic coefficient $C_{w}$. The wings' inertial forces have a small effect because the mass of the insect's wings is less than $5 \%$ of the body's total mass [11]. The unique aerodynamic force, generated by the air pressure on the wing, is applied on its aerodynamic center, which coincides with its center of gravity in practical cases. This center is located at the quarter distance of the wing's chord, from the leading edge, and at 0.6 to 0.7 of the wing's length, measured from the base [11]. The force is perpendicular to the wing's plane and has the opposite direction of the wing's velocity. The module of this force is considered proportional to the square of the wing's velocity relative to the mobile frame $\mathbb{R}^{m}$ attached to the body. This assumption has to be checked on the prototype under construction. The module $f^{w}$ of this force is given by

$$
f^{w}=-\frac{1}{2} \rho C_{w} S_{w} v^{w}\left|v^{w}\right|
$$

$\rho$ is the air density, $S_{w}$ is the wing's surface, $v^{w}$ is the wing's velocity, $C_{w}$ is a coefficient of the aerodynamic force applied on a wing. $C_{w}=C\left(1+C_{f}\right)$ during downstroke and $C_{w}=C\left(1-C_{f}\right)$ during upstroke, where $C \approx 3.5$ is the delayed stall force coefficient derived empirically in [12], [11] and $C_{f}$ is a damping coefficient chosen so that the aerodynamic force is $20 \%$ greater during downstroke than during upstroke. This dissymmetry between the two halfstrokes is explained in [2]. During downstroke, the dorsal side of the wing is opposite to the air flow. Wing base twisting, required for stroke reversal, opposes the ventral side of the wing to the flow. The effective area of the wing is then reduced and the orientation of air circulation about the wing likely reverses, leading to a wing camber alteration, action that is not perfectly achieved by insects. Therefore, downstroke lift is likely to be higher than that of upstroke, so that the averaged force over a single wingbeat period should at least balance the body's weight.

The forces created by left and right wings are projected in the mobile frame $\mathbb{R}^{m}$ and summed up to calculate the global aerodynamic force relative to $\mathbb{R}^{m}$

$$
f^{m}=f_{l}^{m}+f_{r}^{m}
$$

The global torque relative to $\mathbb{R}^{m}$ is defined as the vectorial product of the force $f^{m}$ and the wing's aerodynamic center position $p^{m}$. Angular viscous torques are negligible with respect to aerodynamic torques.

$$
\tau^{m}(t)=p_{l}^{m}(t) \wedge f_{l}^{m}(t)+p_{r}^{m}(t) \wedge f_{r}^{m}(t)
$$

The wing's velocity is the derivative of its center of gravity's position, $v^{m}=\dot{p}^{m} . v^{w}$ is the projection of $v^{m}$ in $\mathbb{R}^{w}$.
The kinematics equation of the quaternion and the attitude dynamics of a rigid body can be written

$$
\begin{aligned}
\dot{P}^{f} & =V^{f} \\
\dot{V}^{f} & =\frac{1}{m} R^{T}(q) f^{m}-c V^{f}-g \\
\left(\begin{array}{c}
\dot{q}_{0} \\
\dot{\vec{q}}
\end{array}\right) & =\frac{1}{2}\left(\begin{array}{c}
-\vec{q}^{T} \\
I_{3} q_{0}+\hat{q}^{T}
\end{array}\right) w^{m} \\
\dot{\omega}^{m} & =J_{m}^{-1}\left(\tau^{m}-\omega^{m} \wedge J_{m} \omega^{m}\right)
\end{aligned}
$$

$P^{f} \in \mathbb{R}^{3}$ and $V^{f} \in \mathbb{R}^{3}$ are respectively the linear position and velocity (relative to the fixed frame $\mathbb{R}^{f}$ ) of the body's center of gravity. $R(q) \in S O(3)=\left\{R(q) \in \mathbb{R}^{3 \times 3}\right.$ : $\left.R^{T}(q) R(q)=I, \operatorname{det} R(q)=1\right\}$ is the rotation matrix as defined in (3). $c$ is the viscous damping coefficient and $g$ the gravity vector. $f^{m} \in \mathbb{R}^{3}$ and $\tau^{m} \in \mathbb{R}^{3}(5,6) . q$ is the quaternion defined in (2). $J_{m} \in \mathbb{R}^{3 \times 3}$ is the inertia matrix of the body relative to $\mathbb{R}^{m}$ and $I_{3}$ is the identity matrix.

\section{Averaged model}

Averaging theory widely studied in [13], [14] can be applied to high frequency systems like flapping UAVs [15], [5].

Theorem 1: [15] Consider a time varying system and its average over a period $T$ :

$$
\begin{aligned}
& \left\{\begin{aligned}
\dot{x} & =f(x, u, \dot{u}) \\
u & =g(v, t) \\
v & =h(x) \\
g(v, t) & =g(v, t+T) \\
\dot{\bar{x}} & =\bar{f}(\bar{x}, \bar{v}) \\
\bar{f}(x, v) & =\frac{1}{T} \int_{0}^{T} f(x, g(v, t), \dot{g}(v, t)) d t \\
\bar{v} & =h(\bar{x})
\end{aligned}\right.
\end{aligned}
$$

where $\{x, \bar{x}\} \in \mathbb{R}^{n}, u \in \mathbb{R}^{m}, v \in \mathbb{R}^{p}$ and all functions and their derivatives are continuous up to the second order. If $\bar{x}=0$ is an exponentially stable equilibrium point for the averaged system (12), then there exists $k>0$ such that $\|x(t)-\bar{x}(t)\|<k T$ for all $t \in[0, \infty)$. Moreover the original system (11) has a unique, exponentially stable, T-periodic orbit $x_{T}(t)$ with the property $\left\|x_{T}(t)\right\|<k T$.

The proof of this theorem is based on dynamical systems of the form $\dot{x}=\epsilon f(x, t, \epsilon)$ [13], where $f$ is $\tilde{T}$-periodic in $t$ and $\epsilon$ a small parameter. Applying the timescale change $\tau=t / T$, (11) can be written $\frac{d x}{d \tau}=T f(x, g(h(x), T \tau), \dot{g}(h(x), T \tau), T \tau)=T \tilde{f}(x, \tau)$, where $\tilde{f}$ is 1 -periodic in its second argument. Identifying to the general form defined above, $T$ is considered as the perturbation parameter $\epsilon$.

$T$ is the wingbeat period, $u=\left(\phi^{l}, \phi^{r}, \psi^{l}, \psi^{r}\right)$ represents the flapping and rotation angles for left and right wings and $v=\left(\phi_{0}^{l}, \phi_{0}^{r}, \psi_{0}^{l}, \psi_{0}^{r}\right)$ the amplitudes of the angles (1), $f(x, u, \dot{u})$ is the system defined by equations (4-10).

As shown in Theorem 1 for high frequency systems, a stabilizing control law of the averaged model (12) will stabilize the time varying model (11) for sufficiently 
high flapping frequency. Therefore, the averaged dynamics of the time varying model, represented by the aerodynamic forces and torques, have been calculated [16], $\left(\bar{f}_{x}, \bar{f}_{z}, \bar{\tau}_{x}, \bar{\tau}_{z}\right)=\Lambda\left(\phi_{0}^{l}, \phi_{0}^{r}, \psi_{0}^{l}, \psi_{0}^{r}\right)$.

\section{Control constraints}

As it will be detailed thereafter, the control law is used to determine the wings angles amplitudes so that the flapping UAV generates averaged aerodynamic forces and torques over a wingbeat period equal to the control forces and torques. The micro UAV is supposed to move in the horizontal plane due to a thrust control force $u_{d}$, vertically due to a lift control force $u_{h}$, and its attitude is stabilized due to the roll, pitch and yaw control torques $\left(\Gamma_{1}, \Gamma_{2}, \Gamma_{3}\right)$. Therefore, $\left(\bar{f}_{x}, \bar{f}_{z}, \bar{\tau}_{x}, \bar{\tau}_{z}\right)=\left(u_{d}, u_{h}, \Gamma_{1}, \Gamma_{3}\right)$. Consequently, the control forces and torques can be expressed as a function of the wings angles amplitudes [16]

$$
\Lambda\left(\phi_{0}^{l}, \phi_{0}^{r}, \psi_{0}^{l}, \psi_{0}^{r}\right)=\left(u_{d}, u_{h}, \Gamma_{1}, \Gamma_{3}\right)
$$

Obviously, the thrust and lift forces and the roll and yaw torques are generated by the movements of the wings. The pitch control torque, $\Gamma_{2}$, will be generated by a small mass moving inside the body, hence changing its center of gravity. Considering that

$$
\begin{aligned}
0 & \leq \phi_{0} \leq \overline{\phi_{0}} \\
-\overline{\psi_{0}} & \leq \psi_{0} \leq \overline{\psi_{0}}
\end{aligned}
$$

for left and right wings, system (13) defines a convex set $\Omega$ in the mean control variables $\left(u_{d}, u_{h}, \Gamma_{1}, \Gamma_{3}\right)$ (see Fig. $4, \Omega_{\Gamma_{1}, \Gamma_{3}}$ is the projection of $\Omega$ on the plane $\left.\left(\Gamma_{1}, \Gamma_{3}\right)\right)$. Therefore, anywhere in the set $\Omega$, there exists a wing configuration $\left(\phi_{0}^{l}, \phi_{0}^{r}, \psi_{0}^{l}, \psi_{0}^{r}\right)$ producing the mean desired forces and torques $\left(u_{d}, u_{h}, \Gamma_{1}, \Gamma_{3}\right)$. By averaging the oscillations due to the beating wings, the system (7-10) can be considered as a time invariant model represented by (12), then has the same behavior as a rigid body subject to external forces and torques. Hence, the averaged state of the time varying model $\bar{x}$ is equivalent to a rigid body state $x^{r b}$.

In this paper, only the attitude stabilization is considered. Flapping and rotation angles are calculated in order to bring the body to the equilibrium, while the lift force is supposed to balance the weight, $u_{h}=m g$, and the thrust force is taken to zero so that no horizontal movement is observed, $u_{d}=0$. As seen before, the flapping UAV can be approximated by a rigid body. Therefore, a state feedback control law, asymptotically stabilizing the rigid body's attitude will be proposed.

$$
\left(0, m g, \Gamma_{1}, \Gamma_{3}\right)=\Gamma\left(x^{r b}\right)=\Gamma(\bar{x})
$$

By inverting (13), (15) can be written

$$
\left(\phi_{0}^{l}, \phi_{0}^{r}, \psi_{0}^{l}, \psi_{0}^{r}\right)=\Lambda^{-1} \Gamma(\bar{x})
$$

Then, $\bar{v}=v$ and $h()=.\Lambda^{-1} \Gamma($.$) in equation (12). This$ control law should respect the saturation set $\Omega_{\Gamma_{1}, \Gamma_{3}}$, insuring that the amplitudes of the wings' angles $\left(\phi_{0}^{l}, \phi_{0}^{r}, \psi_{0}^{l}, \psi_{0}^{r}\right)$ remain within the saturation bounds (14).

\section{AtTITUDE CONTROL}

The goal of this paper being the attitude stabilization of the flapping micro UAV, a control law should be applied in order to drive the body to a desired orientation $q_{d}$, while the angular velocity should vanishes: $q \rightarrow q_{d}, \omega^{m} \rightarrow 0$ as $t \rightarrow \infty$. The desired orientation, representing the roll, pitch and yaw angles of the body, is aligned to the fixed frame $\mathbb{R}^{f}$. Two equilibrium

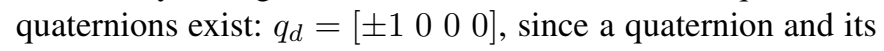
negative represent the same rotation, one of angle $\nu$ and the other of angle $2 \pi-\nu$.

The control torque applied in order to stabilize the attitude of the flapping body was first applied to a rigid body (4 rotors helicopter) in [17]. This control law is extremely simple and therefore suitable for an embedded implementation. Moreover, the control law is robust with respect to aerodynamic coefficient and does not require the knowledge of the inertia. Let $\Gamma=\left[\begin{array}{lll}\Gamma_{1} & \Gamma_{2} & \Gamma_{3}\end{array}\right]^{T}$ be the roll, pitch and yaw control torques.

$$
\Gamma_{i}=-\alpha_{i} \sigma_{M_{2, i}}\left(\lambda_{i}\left[\gamma_{i} \omega_{i}^{r b}+\operatorname{sign}\left(q_{0}^{r b}\right) \sigma_{M_{1, i}}\left(q_{i}^{r b}\right)\right]\right)
$$

It is a bounded state feedback control torque, based in its formulation on the model of a rigid body (equivalent to the averaged model of the flapping body) and applied to the time variant model (flapping micro UAV).

$$
\Gamma_{i}=-\alpha_{i} \sigma_{M_{2, i}}\left(\lambda_{i}\left[\gamma_{i} \bar{\omega}_{i}+\operatorname{sign}\left(q_{0}\right) \sigma_{M_{1, i}}\left(\bar{q}_{i}\right)\right]\right)
$$

where $i \in\{1,2,3\}, \operatorname{sign}\left(q_{0}\right)$ takes into account the possibility of the 2 rotations to drive the body to its equilibrium position; the one of smaller angle is chosen. $\bar{\omega}_{i}$ and $\bar{q}_{i}$ are the averaged angular velocities and quaternion over a single wingbeat period (averaged state $\bar{x}=\left\{\bar{\omega}_{i}, \bar{q}_{i}\right\}, i=1,2,3$ ) representing the time varying angular velocities and quaternion of a rigid body. $\alpha_{i}, \lambda_{i}, \gamma_{i}$ are positive parameters. Differently form [17], $\gamma_{i}$ has been added in order to slow down the convergence of the torque compared to the angular velocity. $\sigma_{M_{1, i}}$ and $\sigma_{M_{2, i}}$ are saturation functions with $M_{1, i}$ and $M_{2, i}$ the saturation bounds: $M_{1, i} \geq 1, M_{2, i} \geq \lambda\left(2 M_{1, i}+\epsilon_{i}\right)$ and $\epsilon_{i}>1$. The $M_{2, i}$ 's are chosen in order to respect input saturations: wings Euler angles and body's length. Based on (13), the maximum flapping and rotation amplitudes, $\bar{\phi}_{0}$ and $\bar{\psi}_{0}$, define a set $\Omega_{\Gamma_{1}, \Gamma_{3}}$ of admissible torques. The saturation bounds $M_{2,1}$ and $M_{2,3}$ are adjusted in (17) so that $\Gamma_{1}$ and $\Gamma_{3}$ remain in the limits of $\Omega_{\Gamma_{1}, \Gamma_{3}}$, which guarantees not to exceed the maximum angles. $M_{2,2}$ should respect the saturation induced by the length of the body, since the pitch torque is generated by a small mass moving inside it.

The asymptotic stability of the closed loop system has been shown in [17] for rigid bodies using the following Lyapunov function (the added parameter $\gamma_{i}$ does not change the proof)

$$
V=\frac{1}{2} \omega^{r b^{T}} J_{m} \omega^{r b}+\kappa\left(\left(1-q_{0}^{r b}\right)^{2}+\vec{q}^{r b^{T}} \vec{q}^{r b}\right)
$$

Therefore, $\bar{\omega} \rightarrow 0$ and $\bar{q} \rightarrow \bar{q}_{d}$ (based on the rigid body case). By means of Theorem $1,\|\omega-\bar{\omega}\|<k_{1} T$ and $\|q-\bar{q}\|<k_{2} T$ for $k_{1,2}>0$ and $T$ the wingbeat period. 


\section{Simulations AND ROBUSTNESS TEST}

The numerical values, used in the simulations of the model proposed in this work, correspond to the Hymenoptera insect [18]. The wingbeat frequency is $100 \mathrm{~Hz}$ and the body mass $500 \mathrm{mg}$. The wing surface $\left(S_{w} \approx 1.5 \mathrm{~cm}^{2}\right)$ is computed so that a vertical ascendant movement can be achieved with a stroke angle remaining lower than $\bar{\phi}_{0}=50^{\circ}$ (maximum stroke amplitude for Hymenoptera). The wing surface corresponds to the physical size of the insect. The rotation angle amplitude is taken to its maximum value $\bar{\psi}_{0}=90^{\circ}$.

Based on these numerical values, the set $\Omega_{\Gamma_{1}, \Gamma_{3}}$ can be determined explicitly. It has been approximated to the largest ellipse $E$ that fits inside $\Omega_{\Gamma_{1}, \Gamma_{3}}$ (see Fig. 4) for calculus simplification reasons. Therefore, the control torques $\Gamma_{1}$ and $\Gamma_{3}$ should respect an ellipsoidal saturation defined by

$$
y^{T} P y=1
$$

where $y=\left(\Gamma_{1} \Gamma_{3}\right)^{T}$ and $P$ is a diagonal definite positive matrix representing the ellipse's semi-axes. Practically, if $\Gamma_{1} \geq \alpha_{1} M_{2,1}, \Gamma_{1}$ could be saturated to $\alpha_{1} M_{2,1}$ and $\Gamma_{3}=0$. To avoid a null yaw control torque in this case, $70 \%$ of $\alpha_{1} M_{2,1}$ will be attributed to $\Gamma_{1}, \Gamma_{3}$ will be calculated by equation (19) defining a set $\Omega_{r}$ (see Fig. 4). This choice is justified by the necessity to bring the UAV to its flat position (horizontal plane) first.

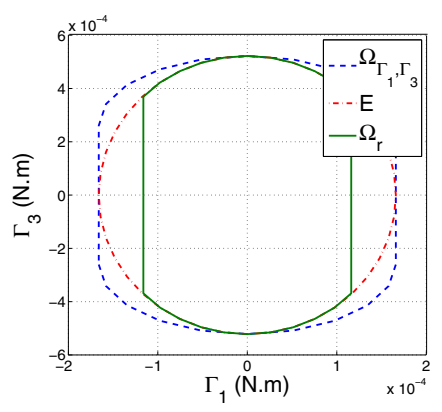

Fig. 4. Yaw torque versus roll torque, defining the saturation set $\Omega_{\Gamma_{1}, \Gamma_{3}}$ approximated to an ellipse $E$ then to a set $\Omega_{r}$.

The stability of the UAV using the proposed control law is shown through simulations. The first one focuses on the convergence of the roll, pitch and yaw angles from an initial attitude $\left(-40^{\circ},-25^{\circ}, 50^{\circ}\right)$ sufficiently far from the origin (see Fig. 5 for the angles, angular velocities and torques convergence). Although the angular velocities seem to be very high, they are comparable with those observed in true insects and with the simulation results of [4]. This is due to the low inertia and the high beating frequency of insects. The desired attitude is reached in a suitable time for practical implementation and comparable to the nature.

The robustness with respect to external disturbances is tested by perturbing the system at $t=2 \mathrm{~s}$. The control torques reach their limits (defined by the bounds as in Fig. 4) to overcome the disturbances, making the flapping UAV execute some rotations before stabilizing. Divergence is then avoided.
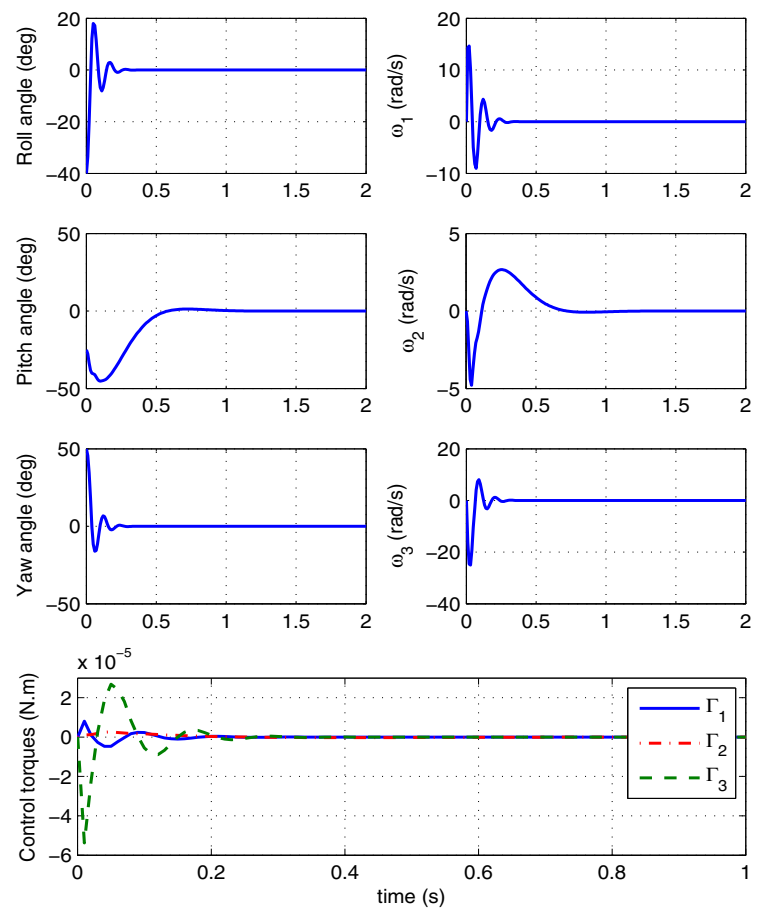

Fig. 5. Convergence of the roll, pitch, yaw angles from initial conditions $\left(-40^{\circ},-25^{\circ}, 50^{\circ}\right)$ (left), angular velocities (right) and zoomed to the $1^{s t} s$ for the control torques of the flapping UAV.

The saturation of the control torques is shown in Fig. 7, that of the angles and the angular velocities in Fig. 6.

Fig. 8 shows the robustness of the control law with respect to a bad estimation of $99.9 \%$ of the aerodynamic coefficient $C_{w}$, known to be difficult to identify. Such a big error is not realistic, since sufficient lift could not be generated to insure the UAV's flight. It is simulated only to emphasize the control law robustness. This property is essential for real time implementation where the flapping UAV can execute missions in different areas having different aerodynamic characteristics.

\section{CONCLUSIONS AND FUTURE WORKS}

The present work deals with the attitude stabilization of a flapping wings UAV. A bounded state feedback control torque, based on the theory of cascade, was applied. This control with low computational cost is suitable for embedded implementation. Even though calculated upon the simplified model, the control strategy can be extended to a more realistic wings parametrization. Robustness with respect to external disturbances and to errors in the estimation of the aerodynamic coefficient are tested. This justifies the use of a simplified model to build the control approach.

The control law will be tested on finer models, specifically the simulator "OSCAB" developed by the ONERA, France. The present work will be extended to 3D movement stabilization. Experimental tests are limited by the development of micro technology to be implemented in $500 \mathrm{mg}$ vehicle. 

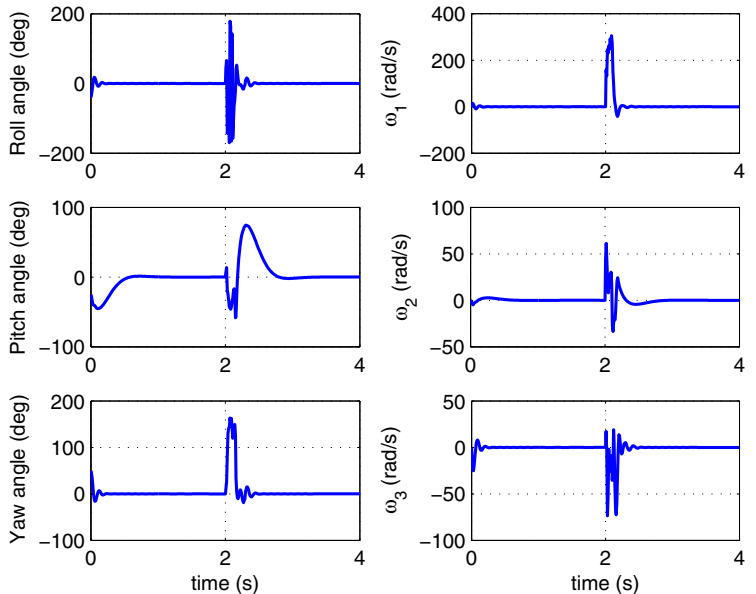

Fig. 6. Robustness wrt to external disturbances applied at $t=2 \mathrm{~s}$ : Convergence of roll, pitch, yaw angles (left) and angular velocities (right) of the flapping UAV.
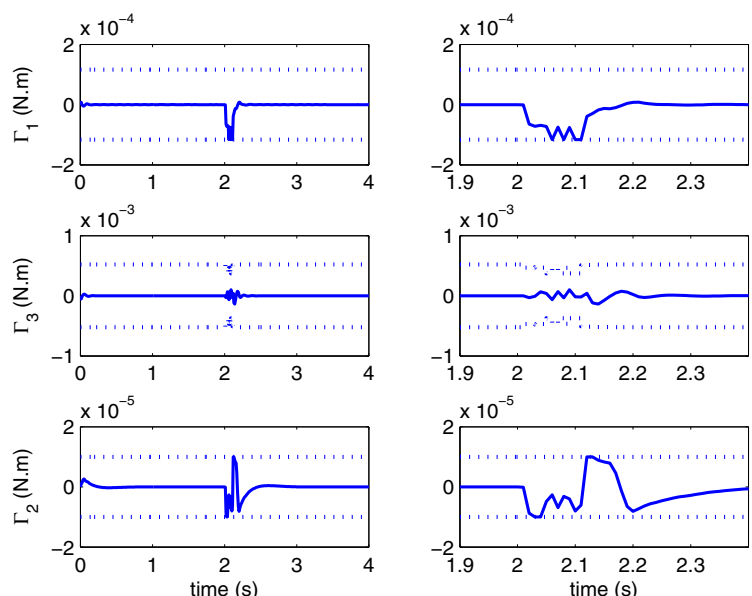

Fig. 7. Robustness wrt to external disturbances applied at $t=2 \mathrm{~s}$ : Convergence of roll, pitch and yaw control torques of the flapping UAV emphasizing saturations. The torques are plotted in continuous line and the bounds in dotted line (left) and zoomed on the saturation (right). The bound on $\Gamma_{3}$ depends on the value of $\Gamma_{1}$ within $\Omega_{r}$ (19).

\section{REFERENCES}

[1] M. S. Triantafyllou and G. S. Triantafyllou, "An efficient swimming machine," Scientific American, vol. 272, no. 3, pp. 64-70, 1995.

[2] R. Dudley, The biomechanics of insect flight: form, function, evolution. Princeton Univerity Press, 2002.

[3] X. Deng, L. Schenato, W.-C. Wu, and S. Sastry, "Flapping flight for biomimetic robotic insects: Part I- system modeling," IEEE Transactions on Robotics, vol. 22, no. 4, August 2006

[4] _ - "Flapping flight for biomimetic robotic insects: Part II- flight control design," IEEE Transactions on Robotics, vol. 22, no. 4, pp. 789-803, August 2006.

[5] T. Rakotomamonjy, "Modelization and flight control of a flapping-wing micro air vehicle," Ph.D. dissertation, University Paul Cézanne Aix Marseille, 2006.

[6] X. Deng, L. Schenato, and S. Sastry, "Model identification and attitude control scheme for a micromechanical flying insect," in 7th International Conference on Control, Automation, Robotic and Vision, Singapore, December 2002, pp. 1007-1012.
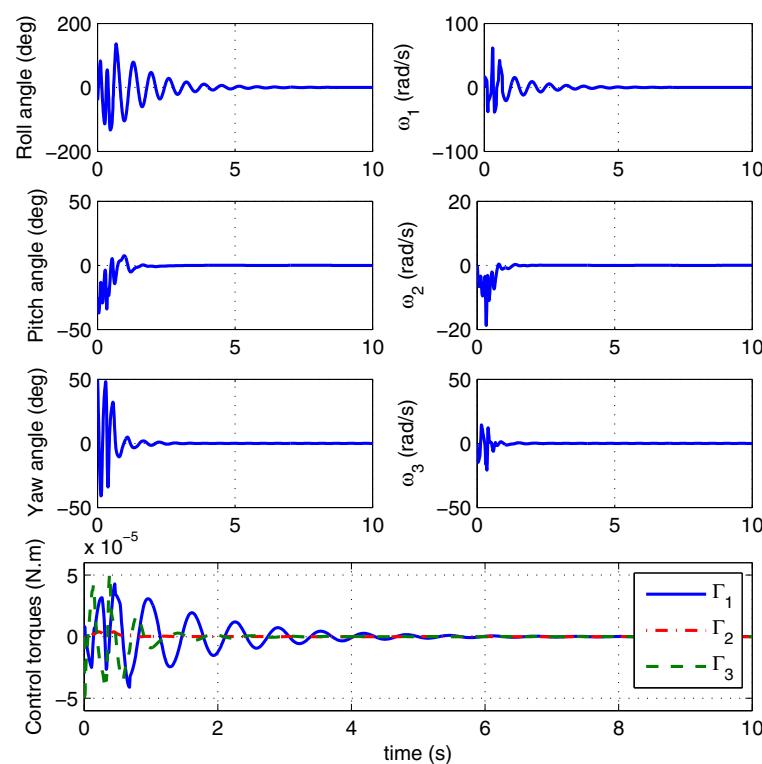

Fig. 8. Robustness wrt aerodynamic coefficient error of $99.9 \%$ : Convergence of roll, pitch, yaw angles (left), angular velocities (right) and control torques (bottom) of the flapping UAV.

[7] L. Schenato, W.-C. Wu, and S. Sastry, "Attitude control for a micromechanical flying insect via sensor output feedback," in 7th International Conference on Control, Automation, Robotic and Vision, Singapore, December 2002, pp. 1031-1036.

[8] _ - "Attitude control for a micromechanical flying insect via sensor output feedback," IEEE Journal of Robotics and Automation, vol. 20, no. 1, pp. 93-106, February 2004.

[9] L. Schenato, X. Deng, and S. Sastry, "Hovering flight for a mocromechanical flying insect: Modeling and robust control synthesis," in 15th IFAC World Congress on Automatic Control, Barcelona, Spain, July 2002.

[10] M. Shuster, "A survey of attitude representations," Journal of astronautical sciences, vol. 41, no. 4, pp. 439-517, 1993.

[11] L. Schenato, D. Campolo, and S. Sastry, "Controllability issues in flapping flight for biomimetic micro aerial vehicles (mavs)," in IEEE International Conference on Decision and Control, Las Vegas, USA, December 2003.

[12] M. Dickinson, F.-O. Lehmann, and S. Sane, "Wing rotation and the aerodyanamic basis of insect flight," Science, vol. 284, no. 5422, pp. 1954-1960, 1999.

[13] H. Khalil, Nonlinear Systems. Maxwell MacMillan International, 1992.

[14] P. A. Vela, "Averaging and control of nonlinear systems," Ph.D. dissertation, California Institute of Technology, 2003.

[15] L. Schenato, "Analysis and control of flapping flight: from biological to robotic insects," Ph.D. dissertation, University of California at Berkeley, 2003.

[16] H. Rifai, N. Marchand, and G. Poulin, "OVMI - Towards a 3D-space flapping flight prameterization," in Proceedings of the $3^{\text {rd }}$ International Conference on Advances in Vehicle Control and Safety, Buenos-Aires, Argentina, 2007, pp. 181-186.

[17] J. Guerrero-Castellanos, A. Hably, N. Marchand, and S. Lesecq, "Bounded attitude stabilization: Application on four rotor helicopter," in Proceedings of the 2007 IEEE International Conference on Robotics and Automation, Roma, Italy, 2007.

[18] C. R. Knospe, "Insect flight mechanisms: Anatomy and kinematics," University of Virginia, Tech. Rep., 1998. 